\title{
SIMPLEXES SELF-POLAR FOR A SIMPLEX
}

\author{
AUGUSTINE O. KONNULLY \\ (Received 28 May 1969; revised 21 July 1969) \\ Communicated by T. E. Room
}

In this paper we prove the existence of simplexes self-polar for a given simplex in projective space of dimensions higher than two. And noting the reciprocity of the relationship between a simplex and any simplex self-polar for it, further, we consider a configuration in projective space associated with a pair of mutually selfpolar simplexes. Finally the results obtained are related to the simplexes determined by Hadamard matrices in space of special dimensions.

\section{Polarity with regard to a simplex}

In projective space of $n$ dimensions, $\Pi_{n}$, let $S$ be the simplex of reference with vertex-vectors $\boldsymbol{e}_{0}, \boldsymbol{e}_{1}, \cdots, \boldsymbol{e}_{n}$ and let $\boldsymbol{a}=a_{0} \boldsymbol{e}_{0}+a_{1} \boldsymbol{e}_{1}+\cdots+a_{n} \boldsymbol{e}_{n}=\left(a_{0}, a_{1}, \cdots\right.$, $a_{n}$ ) be the co-ordinate vector of an arbitrary point disjoint from $S$. The $\Pi_{n-1}$ joining the face $x_{i}=x_{j}=0$ of $S$ to the point $a$ meets the edge $\left\langle\boldsymbol{e}_{i}, \boldsymbol{e}_{j}\right\rangle$ in the point $a_{i} \boldsymbol{e}_{i}+a_{j} \boldsymbol{e}_{j}$. The harmonic conjugate of this point with regard to the vertices $\boldsymbol{e}_{i}$ and $e_{j}$ is $a_{i} e_{j}-a_{j} e_{j} . S$ and $a$ determine $\frac{1}{2} n(n+1)$ such points, all lying in the prime $\alpha \equiv \boldsymbol{a}^{* T} \boldsymbol{x}=0$, where $a_{i}^{*}=a_{i}^{-1}$ and $\boldsymbol{a}^{*}=\left(a_{0}^{*}, a_{1}^{*} \cdots a_{n}^{*}\right)$.

Definition. The point $a$ and the prime $\alpha \equiv a^{* T} x=0$ are designated pole and polar with regard to $S$.

\section{Self-polar simplexes}

Definition. A simplex will be said to be self-polar for a given simplex, if and only if each prime face of it is the polar of the opposite vertex with regard to the given simplex.

Mutually self-polar tetrahedra and mutually self-polar simplexes have been considered by S. R. Mandan [1] and Ashgar Hameed [2].

Let $\boldsymbol{A}$ be a simplex with vertices $\boldsymbol{a}^{0}, \boldsymbol{a}^{1}, \cdots, \boldsymbol{a}^{n}$. If each of its faces is the polar for $S$ of the corresponding vertex, then

$$
\left(a^{i}\right)^{* T} a^{j} \begin{cases}=0 & \text { if } i \neq j \\ =n+1 & \text { if } i=j\end{cases}
$$


Write also $A$ for the matrix of which the columns are the vectors $\boldsymbol{a}^{i}$, that is, $A=\left[\boldsymbol{a}^{0}, \boldsymbol{a}^{1}, \cdots, \boldsymbol{a}^{n}\right]$. Then from the definition it follows that

THEOREM 1. The simplex $A$ is self-polar for the simplex of reference $S$ if and only if

For $n=1$,

$$
A^{* T} A=(n+1) I .
$$

$$
A=\left[\begin{array}{rr}
a^{0} & a^{0} \\
a^{1} & -a^{1}
\end{array}\right],
$$

and the simplex consists of a pair of points harmonically separating the two points of reference. For $n=2$ there is no real matrix with the required property, (1) being seen to imply

$$
\sum_{i=0}^{2}\left(a_{k}^{i} \mid a_{j}^{i}\right)^{2}=0, \quad(k, j=0,1,2 ; k \neq j)
$$

which no set of real numbers can satisfy.

When $n \geqq 3$ we can find real matrices; and correspondingly there exist simplexes self-polar for a simplex. To prove this, let $B$ be a simplex with vertices $\boldsymbol{b}^{0}, \boldsymbol{b}^{1}, \cdots, \boldsymbol{b}^{n} ; \boldsymbol{b}^{0}=(k, 1,1, \cdots, 1), \boldsymbol{b}^{1}=(1, k, 1, \cdots, 1), \cdots, \boldsymbol{b}^{n}=(1,1, \cdots$, $1, k)$. The matrix $B=\left[\boldsymbol{b}^{0}, \boldsymbol{b}^{1}, \cdots, \boldsymbol{b}^{n}\right]$ will satisfy the condition (1) provided

$$
k+\frac{1}{k}+(n-1)=0
$$

If $n \geqq 3$ there is a real value of $k$ which satisfies this equation. If $k_{n}$ denote one of the roots of (2) and $k_{n}^{*}$ its reciprocal then it is clear that $B_{n}$ and $B_{n}^{*}$, obtained from $B$ on putting $k=k_{n}$ of $k_{n}^{*}$, are real matrices satisfying (1), and the simplex with columns of $B_{n}$ or $B_{n}^{*}$ for co-ordinate vectors of the vertices will be self-polar for $S$. When $n=3, k_{n}=k_{n}^{*}$ and $B_{n}=B_{n}^{*}$. Thus there is at least one simplex self-polar for a given simplex $S$, when $n>2$.

It may be now shown that if there is one simplex self-polar for a given simplex $S$, then there are infinitely many simplexes similarly self-polar for $S$. For, if $B$ be a simplex with vertices $\boldsymbol{b}^{0}, \boldsymbol{b}^{1}, \cdots, \boldsymbol{b}^{n}, \boldsymbol{b}^{i}=\left(b_{0}^{i}, b_{1}^{i}, \cdots, b_{n}^{i}\right)$, self-polar for $S$, and if $M$ be the matrix obtained from the matrix $B=\left[b^{0}, b^{1}, \cdots, b^{n}\right]$ by multiplying the rows of $B$ by non-zero real numbers $c_{0}, c_{1}, \cdots, c_{n}$ respectively so that $M=$ $\left[\boldsymbol{m}^{0}, \boldsymbol{m}^{1}, \cdots, \boldsymbol{m}^{n}\right]$, where $\boldsymbol{m}^{i}=\left(c_{0} b_{0}^{i}, c_{1} b_{1}^{i}, \cdots, c_{n} b_{n}\right)$ then

$$
M^{* T} M=B^{* T} B=(n+1) I
$$

Hence the simplex $M$, that is the simplex with the columns of $M$ for co-ordinate vectors of vertices, is self-polar for $S$. The numbers $c_{0}, c_{1}, \cdots, c_{n}$ being arbitrary, given any point not on any one of the faces of $S$, it is always possible to choose them so that $\boldsymbol{m}^{i}$ is this point. That means, given an arbitrary point in $\Pi_{n}$ (not on any one of the faces of $S$ ) there is at least one simplex self-polar for $S$ with this point for a vertex. Thus 
THEOREM 2. In real projective space of 2 dimensions there is no simplex (triangle) self-polar for another simplex (triangle). But there exist infinitely many simplexes self-polar for a given simplex in space of every dimensions higher than 2.

\section{Related self-polar simplexes}

Let $A$ be a simplex self-polar for $S$. Also $A$ be the matrix with the coordinatevectors of the vertices of the simplex for columns. Then from condition (1), since $A^{* T}=A^{T *}$, we deduce

$$
\begin{aligned}
& (n+1) I=A^{* T} A=\left(A^{*}\right)^{T}\left(A^{*}\right)^{*}=\left(A^{*}\right)^{* T} A^{*} \\
& (n+1) I=A A^{* T}=\left(A^{T *}\right)^{T} A^{T}=\left(A^{T}\right)^{* T} A^{T} .
\end{aligned}
$$

So also

$$
\begin{aligned}
(n+1) A^{-1}=A^{T *} \Rightarrow\left(A^{-1}\right)^{*}=(n+1) A^{T} \Rightarrow & \left(A^{-1}\right)^{* T}=(n+1) A \\
& \Rightarrow\left(A^{-1}\right)^{* T} A^{-1}=(n+1) 1 .
\end{aligned}
$$

Hence

THEOREM 3. If the columns of $A$ are the coordinate-vectors of the vertices of a simplex self-polar for $S$, so also are the columns of $A^{*}, A^{T}$ and $A^{-1}$.

The coordinate-vectors of the vertices of $S$ in relation to $A$ as simplex of reference are the columns of $A^{-1}$ so that the relation $\left(A^{-1}\right)^{* T} A^{-1}=(n+1) I$ means that $S$ is self-polar for $A$. So

THEOREM 4. If $A$ is self-polar for $S$, then $S$ is self-polar for $A$.

\section{Simplexes in perspective}

Let $S$ be the simples of reference and $u=\left(u_{0}, u_{1}, \cdots, u_{n}\right)$ be the coordinatevector of any point disjoint from $S$. Any simplex $T_{k}$ in perspective with $S$ from the point $\boldsymbol{u}$ has vertices given by $\boldsymbol{t}_{\boldsymbol{k}}^{0}=\left(k_{0} u_{0}, u_{1}, \cdots, u_{n}\right), \boldsymbol{t}_{\boldsymbol{n}}^{1}=\left(u_{0}, k_{1} u_{1}, u_{2}, \cdots, u_{n}\right)$, $\cdots, t_{k}^{n}=\left(u_{0}, u_{1}, \cdots, k_{n} u_{n}\right) . T_{k}$ is self-polar for $S$ if and only if $T_{k}=\left[t_{k}^{0}, t_{k}^{1}, \cdots\right.$, $\left.t_{k}^{n}\right]$ satisfies the condition (1), that is, $T_{k}^{* T} T_{k}=(\mathrm{n}+1) I$. This requires that $k_{0}=k_{1}$ $=\cdots=k_{n}=k$, where $k+1 / k+(n-1)=0$, that is, $k=k(1,1, \cdots, 1), k=k_{n}$ or $k_{n}^{*}$. If $n>3, k_{n}$ and $k_{n}^{*}$ are distinct, and if $n=3$, they are equal. Thus

THEOREM 5. Given any simplex $S$ and any point $\boldsymbol{u}$ disjoint from $S$, there are two simplexes perspective with $S$ from $\boldsymbol{u}$ and self-polar for $S$, if $\boldsymbol{n}>3$.

The system $S, \boldsymbol{u}, T_{\boldsymbol{k}}, \boldsymbol{k}=k(1,1, \cdots, 1)$, determines a central collineation (homology) with centre $\boldsymbol{u}$ and axial prime $\boldsymbol{u}^{* T} \boldsymbol{x}=0$ which maps $S$ to $T$. Let $\boldsymbol{u}$, $a^{1}, a^{2}, \cdots, a^{n}$ be the vertices of a simplex self-polar for $S$ so that, in particular, each point $\boldsymbol{a}^{i}$ lies in the axial plane $\boldsymbol{u}^{* T} \boldsymbol{x}=0$ and each prime $\left(\boldsymbol{a}^{i}\right)^{* T} \boldsymbol{x}=0$ passes through the centre $u$. Under this homology $S$ maps to the simplex $T_{k}$ and polar 
pairs for $S$ to the polar pairs for $T_{k}$. All points $a^{i}$ are invariant since they lie in the axial prime and all primes $\left(a^{i}\right)^{* T} x=0$ are invariant because they pass through the centre. Thus

THEOREM 6. If two mutually self-polar simplexes $S$ and $T$ are in perspective from a point $\boldsymbol{u}$, then, if any simplex of which $\boldsymbol{u}$ is a vertex is self-polar for $S$, it is selfpolar for $T$ also.

\section{A configuration}

Let $S$ be the simplex of reference and $A$ be any simplex self-polar for $S$ with vertices $\boldsymbol{a}^{0}, \boldsymbol{a}^{1}, \cdots, \boldsymbol{a}^{n}, \boldsymbol{a}^{i}=\left(a_{0}^{i}, a_{1}^{i}, \cdots, a_{n}^{i}\right)$. Let $A^{j}(k)$ denote the simplex with vertices $c_{0}^{j}(k)=\left(k a_{0}^{j}, a_{1}^{j}, \cdots, a_{n}^{j}\right), c_{1}^{j}(k)=\left(a_{0}^{j}, k a_{1}^{j}, a_{2}^{j}, \cdots, a_{n}^{j}\right), \cdots, c_{n}^{j}=\left(a_{0}^{j}\right.$, $\left.a_{1}^{j}, \cdots, k a_{n}^{j}\right) . A^{j}(k)$ is a simplex perspective with $S$ from the point $a^{j}$ which is a vertex of $A$. If $k=k_{n}$ or $k_{n}^{*}, A^{j}(k)$ will be also self-polar for $S$. Then since $A$ is selfpolar for $S$ and has the point $a^{j}$ for a vertex, $A$ will be self-polar for $A^{j}(k)$ also. Thus $S, A, A^{j}(k)$ form a set of three mutually self-polar simplexes. And $A^{j}(k)$, $(j=0,1, \cdots, n)$ form a set of $(\mathrm{n}+1)$ sim plexes each self-polar for both $A$ and $S$.

Similarly let $S_{i}(k)$ denote the simplex with $c_{i}^{0}(k), c_{i}^{1}(k) \cdots c_{i}^{n}(k)$ for vertices. Then $S_{i}(k)$ will be a simplex perspective with $A$ from the point $e_{i}^{i}$ which is a vertex of the simplex $S . S$ and $S_{i}(k)$ are mutually self-polar. Writing down the co-ordinate vectors of the vertices of $S_{i}(k)$ in relation to $A$ as simplex of reference and using the condition (1), it is seen that $S_{i}(k)$ is self-polar for $A$ also, if, and only if, $k=k_{n}$ or $k_{n}^{*}$. Thus $S_{i}(k),(i=0,1, \cdots, n)$ are simplexes self-polar for both $S$ and $A$, provided $k=k_{n}$ or $k_{n}^{*}$.

The $(n+1)^{2}$ points which form the vertices of the $(n+1)$ simplexes $A^{j}(k)$ are the same as the $(n+1)^{2}$ points which form the vertices of the $(n+1)$ simplexes $S_{i}(k)$. The simplex $A^{j}(k)$ has one vertex in common with each of the simplexes $S_{0}(k), S_{1}(k), \cdots, S_{n}(k)$. And the simplex $S_{i}(k)$ has one vertex in common with each of the simplexes $A^{j}(k)$.

Since when $k=k_{n}$ or $k_{n}^{*}$ the simplexes $A^{j}(k)$ and $S_{i}(k)$ are self-polar for both $S$ and $A$ the polar of each point $c_{i}^{j}(k)$ is the same for both $S$ and $A$. As each point $c_{i}^{j}(k)$ is the vertex common to the two simplexes $A^{j}(k)$ and $S_{i}(k)$, so also the polar of $c_{i}^{j}(k)$ for $S$ and $A$ will be a prime face common to the two simplexes.

Let $p_{i}^{j}(k)$ denote the polar of $c_{i}^{j}(k)$ for $S$ and $A$. The $(n+1)^{2}$ points $\boldsymbol{c}_{i}^{j}(k)$ and the $(n+1)^{2}$ primes $p_{i}^{j}(k)$ now form a configuration with each point $c_{i}^{j}(k)$ lying in $2 n$ primes and each prime containing $2 n$ points. The point $c_{i}^{j}(k)$ lies in every one of the prime faces of the two simplexes $A^{j}(k)$ and $S_{i}(k)$ except the common face $p_{i}^{j}(k)$; and the prime $p_{i}^{j}(k)$ contains every vertex of the simplexes except the common vertex $\boldsymbol{c}_{i}^{j}(k)$.

For each value of $k=k_{n}, k_{n}^{*}$, there is one such configuration. And the whole configuration for each value, made up of $(n+1)^{2}$ points and $(n+1)^{2}$ primes is selfpolar for both $S$ and $A$. 


\section{In $2^{m}-1$ dimensions}

Consider the space $\Pi_{n}$ where $n$ is a Mersenne number, $n=2^{m}-1$. The $2^{n}$ points $(1, \pm 1, \cdots, \pm 1)$ in $\Pi_{n}$ form a set $A$ called a set of associated points. The simplex of reference is called the diagonal simplex of the set. We prove that the points of the set fall into the vertices of a number of simplexes each of which is self-polar for the diagonal simplex.

Let

$$
\begin{gathered}
U_{1}=\left[\begin{array}{rr}
1 & 1 \\
1 & -1
\end{array}\right], \quad U_{2}=\left[\begin{array}{rr}
U_{1} & U_{1} \\
U_{1} & -U_{1}
\end{array}\right], \cdots \\
U_{m}=\left[\begin{array}{rr}
U_{m-1} & U_{m-1} \\
U_{m-1} & -U_{m-1}
\end{array}\right] .
\end{gathered}
$$

Then $U_{m}$ is a $2^{m} \times 2^{m}$ matrix with the coordinates of some point of the set $A$ in each column.

It is easily verified that the simplex $U_{m}$, that is, the simplex with the columns of $U_{m}$ as coordinate-vectors of vertices, is self-polar for the diagonal simplex $S$. (It may be observed that in each of the matrices $U_{1}, U_{2}, \cdots, U_{m}$, the elements of any column multiplied by the corresponding elements of another column give a column different from the first column of the matrix and that the sum of the elements of every column other than the first column is zero; this, if true in $U_{r}$, being easily seen to be true in $U_{r+1}$.) If the rows of $U_{m}$ are multiplied respectively by $a_{0}, a_{1}, \cdots, a_{n}$ where $a_{i}$ are all \pm 1 or the coordinates of any one of the points of set $A$, we will have a simplex which is self-polar for $S$ and has all its vertices in the set $A$.

The set $G$ of all $(n+1)$-tuples $\left(a_{0}, a_{1}, \cdots, a_{n}\right)$ where $a_{0}=1, a_{i}= \pm 1$, $(i=1,2, \cdots, n)$, with the law of composition defined on it by $\left(a_{0}, a_{1}, \cdots, a_{n}\right)$. $\left(b_{0}, b_{1}, \cdots, b_{n}\right)=\left(a_{0} b_{0}, a_{1} b_{1}, \cdots, a_{n} b_{n}\right)$ is a group with $(1,1, \cdots, 1)$ for unit element. And the subset $H$ of the $(n+1)$ elements $\left(u_{0}, u_{1}, \cdots, u_{n}\right)$ where $u_{0}, u_{1}, \cdots$ $u_{n}$ are the elements of a column of $U_{m}$ is a subgroup of $G$, the product of any two elements of the set $H$ being always an element of the set. Let $H, H_{1}, \cdots, H_{k}$ be the distinct cosets of $H$ in $G$.

Now there is a one-to-one correspondence between $G$ and the set $A$ of points. To $H$ corresponds the set of points which form the vertices of the simplex $U_{m}$; and to each $H_{i}$ corresponds a simplex $U_{m}^{i}$ obtained by multiplying the rows of $U_{m}$ respectively by the coordinates of one of the points of $A$. As $U_{m}$ is self-polar for $S$, so each simplex $U_{m}^{i}$ is self polar for $S$. And as $G$ is partitioned into the classes $H, H_{1}, \cdots, H_{k}$ so that each element of $G$ belongs to one and only one of these classes so also the points of the set $A$ are partitioned into the vertices of the simplexes $U_{m}, U_{m}^{1}, U_{m}^{2}, \cdots, U_{m}^{k}$. Thus the points of the set $A$ fall into a number of simplexes each of which is self-polar for $S$. The number of these simplexes is evidently $2^{n} /(n+1)=2^{n-m}$. 
If the elements of the $(j+1)$-th row of $U_{m}^{i}$ are all multıplied by $(-1)$, we will have a simplex self-polar for $S$ and one among the simplexes into which the points of the set $\boldsymbol{A}$ fall. This simplex will be prespective with $U_{m}^{i}$ from the $(j+1)$-th vertex of $S$, i.e. the point $e_{j}$. As $j$ ranges over $0,1,2, \cdots, n$, each of the $(n+1)$ simplexes we get is prespective with $U_{m}^{i}$ from one of the vertices of $S$. Thus the simplexes $U_{m}, U_{m}^{1}, \cdots, U_{m}^{k}$ are such that each one is perspective with $(n+1)$ others, the centres of perspective being the different vertices of $S$.

In particular, when $n=3$ the set of associated points fall into two tetrahedra

$$
\left[\begin{array}{rrrr}
1 & 1 & 1 & 1 \\
-1 & -1 & 1 & 1 \\
-1 & 1 & -1 & 1 \\
-1 & 1 & 1 & -1
\end{array}\right] \text { and }\left[\begin{array}{rrrr}
1 & 1 & 1 & 1 \\
1 & 1 & -1 & -1 \\
1 & -1 & 1 & -1 \\
1 & -1 & -1 & 1
\end{array}\right]
$$

which are both self-polar for the tetrahedron of reference. They are also self-polar for each other. The desmic system thus consists a set of three tetrahedra which are mutually self-polar.

My thanks are due to the referee for the present form of the paper.

\section{References}

[1] S. R. Mandan, 'Properties of Mutually Self-polar Tetrahedra', Bull. Ca. Math. Soc. 33 (1941), $147-155$.

[2] Asghar Hameed, 'On Mutually Self-polar Simplexes', Bull. Ca. Math. Soc. 35 (1943), 43.

St. Albert's College, Ernakulam

Cochin, India 